\title{
EIGENVALUE APPROXIMATION BY A MIXED METHOD FOR RESONANT INHOMOGENEOUS CAVITIES WITH METALLIC BOUNDARIES
}

\author{
VINCENT LEVILLAIN
}

\begin{abstract}
For an inhomogeneous cavity bounded by a perfect conductor, we prove that the approximation of the eigenvalues for the Maxwell problem leads to a second-order rate of convergence when using mixed finite elements. If the cavity has a disconnected boundary, the problem has null eigenvalues. We verify the existence of null eigenvalues for the approximate problem. They do not mix with the others that still converge at the same rate.
\end{abstract}

\section{INTRODUCTION}

The approximation of eigenvalues involves problems that are of great interest. We mention, for example, the works of Osborn [10], Mercier, Osborn, Rappaz, and Raviart [8], and Brezzi and Raviart [5]. More specifically, mixed methods are used for this purpose (see Ishihara [7]). The aim of this paper is to apply results of Mercier, Osborn, Rappaz, and Raviart [8] on eigenvalues, and of Nedelec [9] on mixed finite elements, to prove a second-order rate of convergence for the harmonic Maxwell equation.

Another part of the paper concerns cavities with disconnected boundaries. The operator of the problem is then no longer elliptic. Work on this kind of geometry can be found in Bossavit [3], and in Bendali, Dominguez, and Gallic [2].

First, we deal with a connected boundary to get an elliptic problem, which is necessary in order to use known results. Then we extend the work to disconnected boundaries and prove that the zero eigenvalues of the curl operator have approximate equivalents that do not mix with the other converging eigenvalues.

To conclude, we show numerical experiments on a cube, for which eigenvalues are known analytically.

\section{THE CONTINUOUS PROBLEM}

Let $\Omega$ be a bounded cavity, with a connected boundary, filled with an inhomogeneous medium. We consider the following problem:

Received December 18, 1989; revised January 22, 1991.

1991 Mathematics Subject Classification. Primary 65N30. 


$$
\left\{\begin{array}{l}
\text { Find }(E, H) \in H(\operatorname{curl}, \Omega) \text { satisfying } \\
\operatorname{curl} E+i \omega H=0 \\
\operatorname{curl} H-i \omega E=0 \\
\operatorname{div}(\mu H)=0,\left.H \cdot n\right|_{\partial \Omega}=0 \\
\operatorname{div}(\varepsilon E)=0, E \times n \mid \partial \Omega=0
\end{array}\right.
$$

where $\varepsilon$ and $\mu$ are strictly positive bounded real functions. Let $E^{\prime}$ be a test function belonging to $H(\mathrm{Curl}, \Omega)$, satisfying $E^{\prime} \times n=0$ on the boundary $\partial \Omega$. Then applying the Green formula

$$
\int_{\Omega} \operatorname{curl} H \cdot \bar{E}=\int_{\Omega} H \cdot \operatorname{curl} \bar{E}-\int_{\partial \Omega}(\bar{E} \times n) \cdot H
$$

to the first system, we obtain the variationally posed problem:

$$
\int_{\Omega} \mu^{-1} \operatorname{curl} E \cdot \operatorname{curl} \bar{E}^{\prime}-\omega^{2} \int_{\Omega} \varepsilon E \cdot \bar{E}^{\prime}=0 .
$$

Given two regular complex functions $\varepsilon$ and $\mu$, with strictly positive bounded real parts, this is an eigenvalue problem in a variational form:

$$
\left\{\begin{array}{l}
\text { Find } E \in H(\operatorname{curl}, \Omega), E \times\left. n\right|_{\partial \Omega}=0, \operatorname{div} \varepsilon E=0, \lambda \in R \\
\text { such that } \forall E^{\prime} \in H(\operatorname{curl}, \Omega) \text { with } E^{\prime} \times n=0, \\
\int_{\Omega} \mu^{-1} \operatorname{curl} E \cdot \operatorname{curl} \bar{E}^{\prime}=\lambda \int_{\Omega} \varepsilon E \cdot \bar{E}^{\prime} .
\end{array}\right.
$$

Therefore, we introduce the following spaces:

$$
\begin{aligned}
W & =H_{0}^{1}(\Omega), \\
X & =\left\{E \in L^{2}(\Omega)^{3}, \operatorname{curl} E \in L^{2}(\Omega)^{3}, E \times\left. n\right|_{\partial \Omega}=0\right\}, \\
H & =L^{2}(\Omega)^{3}
\end{aligned}
$$

and the continuous bilinear forms

$$
\begin{aligned}
a: X \times X & \rightarrow C \\
\left(E, E^{\prime}\right) & \rightarrow \int_{\Omega} \frac{1}{\mu} \operatorname{rot} E \cdot \operatorname{rot} \bar{E}^{\prime}+\int_{\Omega} \varepsilon E \cdot \bar{E}^{\prime}, \\
b: X \times W & \rightarrow C \\
(E, q) & \rightarrow \int_{\Omega} \varepsilon E \cdot \operatorname{grad} \bar{q} \\
r: H \times H & \rightarrow C \\
\left(E, E^{\prime}\right) & \rightarrow 2 \cdot \int_{\Omega} \varepsilon E \cdot \bar{E}^{\prime}
\end{aligned}
$$

that satisfy the properties:

$\mathrm{H} 1: r$ is continuous and bounded on $H \times H$,

$\mathrm{H} 2: b(v, q)=0$ for every $v$ in $X$ implies $q=0$.

(Simply consider $v=\operatorname{grad} q$ that belongs to $X$ : since $\varepsilon$ and $\mu$ have strictly positive bounded real parts, we apply the Poincaré inequality to conclude that $\operatorname{grad} q=0$ implies $q=0$ in $\Omega$.) 
Let us now introduce the following problem: with $g$ given in $H$, find $(E, p) \in X \times W$ such that, $\forall\left(E^{\prime}, p^{\prime}\right) \in X \times W$ :

$$
\left\{\begin{aligned}
a\left(E, E^{\prime}\right)+\bar{b}\left(E^{\prime}, p\right) & =r\left(g, E^{\prime}\right), \\
b\left(E, p^{\prime}\right) & =0 .
\end{aligned}\right.
$$

We have

Lemma 1.1. For each $g$ given in $H$, there is a unique solution $(E, p)$ of problem (II) that depends continuously on $g$. As $g$ belongs to $H$, we have the higher regularity:

$$
\|E\|_{H^{2}}+\|p\|_{H_{0}^{1}} \leq c\|g\|_{L^{2}} .
$$

Furthermore, if $\operatorname{div} E \in L^{2}$, then $p$ belongs to $H^{2}$, and we have

$$
\|E\|_{H^{2}}+\|p\|_{H^{2}} \leq c\left(\|g\|_{L^{2}}+\|\operatorname{div} g\|_{L^{2}}\right) .
$$

Proof. The bilinear form $a$ is $V$-elliptic: it is equivalent to the $H$ (curl) norm, since $\varepsilon$ and $\mu$ have positive real parts. We have the following property:

H3: $b$ satisfies the Brezzi-Babuška condition (cf. Brezzi [4])

$$
\inf _{\|q\| \neq 0} \sup _{\|E\| \neq 0} \frac{|b(E, q)|}{\|E\| \cdot\|q\|}>0 .
$$

Indeed, consider $u=\operatorname{grad}(q)$; since the seminorm of the gradient is equivalent to the norm, the inequality is verified. Brezzi's result [4] now gives us the existence and uniqueness of $(u, p)$. The first regularity inequality is a classical result that can be found in Agmon, Douglis, and Nirenberg [1]. Finally, if $\operatorname{div} g \in L^{2}$, then $\Delta p \in L^{2}$, and so $p \in H^{2}$.

\section{THE APPROXIMATE PROBLEM}

We define a triangulation of the domain $\Omega$, with tetrahedra, and to avoid the problem of volume and surface approximation, we will suppose that $\Omega$ is a polyhedral open set. We define the spaces

$$
\begin{aligned}
X_{h} & =\left\{E \in H(\operatorname{rot}, \Omega), \forall K \in \mathscr{T}_{h},\left.E\right|_{K}(\vec{r})=\vec{\alpha}+\vec{\beta} \times \vec{r}, E \times\left. n\right|_{\partial \Omega}=0\right\}, \\
W_{h} & =\left\{q \in H_{0}^{1}(\Omega),\left.q\right|_{k} \in P^{1}\right\}, \\
V_{h} & =\left\{E_{h} \in X_{h}, b\left(E_{h}, q_{h}\right)=0, \forall q_{h} \in W_{h}\right\} .
\end{aligned}
$$

The system that is numerically solved is

$$
\left\{\begin{array}{l}
\text { Find } E \in X_{h} \text { and } \lambda \in C \text { such that } \forall E^{\prime} \in X_{h} \\
\int_{\Omega} \mu^{-1} \operatorname{curl} E \cdot \operatorname{curl} \bar{E}^{\prime}=\lambda \int_{\Omega} \varepsilon E \cdot \bar{E}^{\prime} .
\end{array}\right.
$$

We have introduced $V_{h}$ in order to be sure that the solutions of the eigenproblem associated with (II.a) below are also solutions of (E). Therefore, we associate the following approximate problem:

With $g$ given in $L^{2}$, find $\left(E_{h}, p_{h}\right)$ in $X_{h} \times W_{h}$ such that for all $\left(E_{h}^{\prime}, p_{h}^{\prime}\right)$ in $X_{h}, W_{h}$ we have

$$
\begin{aligned}
a\left(E_{h}, E_{h}^{\prime}\right)+\bar{b}\left(E_{h}^{\prime}, p_{h}\right) & =r\left(g, E_{h}^{\prime}\right), \\
b\left(E_{h}, p_{h}^{\prime}\right) & =0 .
\end{aligned}
$$


We now have

Lemma 2.1. If $q$ is an element of $W_{h}$, then $\operatorname{grad}(q)$ belongs to $X_{h}$. Proof. Nedelec [9]

The approximate problem satisfies properties $\mathrm{H} 1, \mathrm{H} 2$, and $\mathrm{H} 3$ : indeed, the bilinear form $a$ is $V_{h}$-elliptic because $V_{h}$ is contained in $H$ (curl). Furthermore, Lemma 2.1 implies that the Brezzi-Babuška condition and property $\mathrm{H} 2$ hold.

This leads to

Lemma 2.2. For each $g$ given in $H$, there is a unique solution $\left(E_{h}, p_{h}\right)$ in $X_{h} \times W_{h}$ of problem (II.a) that depends continuously on $g$. The continuity constants do not depend on $h$.

Remark. A part of the set of eigenvectors given by the variational formulation (E) is composed of curl-free functions that do not belong to $V_{h}$ and that are exactly the gradients of $W_{h}$ functions, and we know the dimension of this subspace $\left(N_{s}\right.$, number of vertices inside $\Omega$ ). They are associated with $N_{s}$ zero eigenvalues. We will prove that each of the other eigenvalues converges toward the corresponding eigenvalue of the continuous problem $(\mathrm{P})$, and we will establish the rate of convergence.

\section{RATE OF CONVERGENCE}

Taking into account the preceding paragraphs, we introduce the following definitions:

$$
\begin{aligned}
A: H & \rightarrow X \\
g & \rightarrow E, \\
B: H & \rightarrow W \\
g & \rightarrow p,
\end{aligned}
$$

where $(E, p)$ is the solution of (II) for a given $g$, and

$$
\begin{aligned}
A_{h}: H & \rightarrow X_{h} \\
g & \rightarrow E_{h}, \\
B_{h}: H & \rightarrow W_{h} \\
g & \rightarrow p_{h},
\end{aligned}
$$

where $\left(E_{h}, p_{h}\right)$ is the solution of (II.a) for a given $g$. We also introduce the dual operators $A^{*}, B^{*}, A_{h}^{*}, B_{h}^{*}$, by means of the solutions of the two problems:

$$
\begin{aligned}
a\left(v, A^{*} g\right)+b\left(v, B^{*} g\right) & =r(v, g) \quad \forall v \in X, \\
b\left(A^{*}, g, q\right) & =0 \quad \forall q \in W, \\
a\left(v_{h}, A_{h}^{*} g\right)+b\left(v_{h}, B_{h}^{*} g\right) & =r\left(v_{h}, g\right) \quad \forall v_{h} \in X_{h}, \\
b\left(A_{h}^{*}, g, q\right) & =0 \quad \forall_{q h} \in W_{h} .
\end{aligned}
$$

These "starred" operators satisfy the same regularity results as $A, B$.

We also assume that as $h$ decreases:

$(\mathrm{H}): \sup _{k}(\operatorname{diam}(K)) \leq c \inf _{K}(\operatorname{diam}(K))$, in order to use the following theorem. 
Theorem 3.1. Under hypothesis $(\mathrm{H})$, and if the solutions are sufficiently regular, there exists $c \geq 0$ such that for all $h$

$$
\left\|A g-A_{h} g\right\|_{X}+\left\|B g-B_{h} g\right\|_{W} \leq c \cdot\left(\|A g\|_{H^{2}}+\|B g\|_{H^{2}}\right) .
$$

Proof. Nedelec [9] and Brezzi [4].

We define a norm for the operators,

$$
\|A\|_{X Y}=\sup _{g \in X, g \neq 0} \frac{\|A g\|_{Y}}{\|g\|_{X}} .
$$

We recall some results about the eigenproblem: $A$ is a compact operator from $L^{2}$ in $L^{2}$, so its spectrum consists of a countable set of complex numbers, and each nonzero number is an isolated eigenvalue. Let $\mu$ be a nonzero eigenvalue; then there exists $n_{\alpha}$ such that

$$
\operatorname{Ker}(A-\alpha I)^{n_{\alpha}-1} \neq \operatorname{Ker}(A-\alpha I)^{n_{\alpha}}=\operatorname{Ker}(A-\alpha I)^{n_{\alpha}+1} .
$$

Set $E_{\alpha}=\operatorname{Ker}(A-\alpha I)^{n_{\alpha}}$, with $\operatorname{dim} E_{\alpha}=m$ the algebraic multiplicity of $\alpha$. We verify that $\bar{\alpha}$ is an eigenvalue of $A^{*}$ with the same multiplicity, and we let $E_{\alpha}^{*}$ be the set of the associated generalized eigenvectors.

Another result that we need is a theorem proved by Mercier, Osborn, Rappaz, and Raviart [8]. A version adapted to our problem is

Theorem 3.2. Under the hypotheses $\mathrm{H} 1, \mathrm{H} 2$, and $\mathrm{H} 3$ for both the continuous and the approximate problems, and assuming both $\lim \left\|A-A_{h}\right\|_{H H}=0$ and the continuous dependence of $E_{h}, E, p_{h}, p$ with respect to $g$, we have the following. Let $\alpha$ be a nonzero eigenvalue of $A$ with multiplicity $m$; then for $h$ small enough, exactly $m$ eigenvalues of $A_{h}$ converge to $\alpha$. Furthermore, let $\beta=1 / \alpha$ and $\beta_{i, h}=1 / \alpha_{i, h}$; then there exists $C_{\beta}$ such that

$$
\begin{aligned}
\left|\beta-\frac{1}{m} \sum_{1 \leq j \leq m} \beta_{j, h}\right| \leq C_{\beta} \cdot & \left(\left\|\left(A-A_{h}\right)_{E_{\alpha}}\right\|_{H X} \cdot\left\|\left(A^{*}-A_{h}^{*}\right)_{E_{\alpha}^{*}}\right\|_{H X}\right. \\
+ & \left\|\left(A^{*}-A_{h}^{*}\right)_{E_{h}^{*}}\right\|_{H X} \cdot\left\|\left(B-B_{h}\right)_{E_{\alpha}}\right\|_{H W} \\
& \left.+\left\|\left(A-A_{h}\right)_{E_{\alpha}}\right\|_{H X} \cdot\left\|\left(B^{*}-B_{h}^{*}\right)_{E_{\alpha}^{*}}\right\|_{H W}\right) .
\end{aligned}
$$

Proof. Mercier, Osborn, Rappaz, and Raviart [8].

In order to prove our main result, we need the following lemma.

Lemma 3.3. There holds $\lim _{h \rightarrow 0}\left\|A-A_{h}\right\|_{H H}=0$.

Proof. Let $g$ be given in $L^{2}$, $\|g\|=1$. If $\operatorname{div} g$ is in $L^{2}$, we know that $\lim \left\|A g-A_{h} g\right\|_{H H}=0$, but not in the general case.

Let $\varepsilon \geq 0$; since $A g$ belongs to $H^{2}$ and satisfies $b(A g, q)=0$ for all $q$, we know that there exists $h_{2}$ such that for all $h \leq h_{2}$, there exists $w_{h} \in X_{h}$ such that

We have

$$
b\left(w_{h}, q_{h}\right)=0 \quad \forall q_{h} \in W_{h}, \quad\left\|A g-w_{h}\right\|_{X} \leq \frac{\varepsilon}{3} .
$$

$$
\begin{aligned}
a\left(A_{h} g\right. & \left.-w_{h}, A_{h} g-w_{h}\right) \\
= & -a\left(w_{h}, A_{h} g-w_{h}\right)+a\left(A_{h} g, A_{h} g-w_{h}\right) \\
= & -a\left(w_{h}, A_{h} g-w_{h}\right)+a\left(A g, A_{h} g-w_{h}\right) \\
& +b\left(A_{h} g-w_{h}, B g\right)-b\left(A_{h} g-w_{h}, B_{h} g\right) \\
= & a\left(A g-w_{h}, A_{h} g-w_{h}\right)+b\left(A_{h} g-w_{h}, B g-B_{h} g\right),
\end{aligned}
$$


and also $b\left(w_{h}, q_{h}\right)=0$ and $b\left(A_{h} g, q_{h}\right)=0$ for all $q_{h}$ in $W_{h}$, so we can replace $B_{h} g$ with any $q_{h}$ in $W_{h}$. Since $a$ is elliptic, continuous, and $b$ is continuous, we have

$$
\left\|A_{h} g-w_{h}\right\|_{X}^{2} \leq c\left(\left\|A g-w_{h}\right\|_{W}\left\|A_{h} g-w_{h}\right\|_{W}+\left\|A_{h} g-w_{h}\right\|_{0}\left\|B g-q_{h}\right\|_{1}\right) .
$$

Since $B g$ is in $H^{1}$, there exists $\phi$ in $H^{2}$ such that

$$
\|B g-\phi\|_{1} \leq \frac{\varepsilon}{6},
$$

using the dense inclusion of $H^{2}$ in $H^{1}$, and we can fix $h_{1} \geq 0$ such that

$$
\forall h \leq h_{1}, \exists q_{h} \in W_{h},\left\|\phi-q_{h}\right\|_{1} \leq \frac{\varepsilon}{6},
$$

using the interpolation property of the $H^{2}$ elements by $W_{h}$. So,

$$
\left\|B g-q_{h}\right\|_{1} \leq \frac{\varepsilon}{6}
$$

and finally

$$
\begin{gathered}
\exists h_{3}, \forall h \leq h_{3},\left\|A_{h} g-w_{h}\right\|_{W} \leq \frac{2 c \varepsilon}{3}, \\
\left\|A g-A_{h} g\right\|_{W} \leq \frac{(2 c+1) \varepsilon}{3} .
\end{gathered}
$$

Therefore,

$$
\lim _{h \rightarrow 0}\left\|A g-A_{h} g\right\|_{W}=0 .
$$

Since the unity sphere of $L^{2}$ is a compact set, we finally have

$$
\lim _{h \rightarrow 0}\left\|A-A_{h}\right\|_{H X}=0 \text {, }
$$

which is more than we need.

We can finally prove the following theorem.

Theorem 3.4. Let $\lambda$ be an eigenvalue for problem $(\mathrm{P})$ with algebraic multiplicity $m$. Then for $h$ small enough, $m$ eigenvalues $\lambda_{i, h}$ of the approximate problem (E) converge towards $\lambda$ and there exists $c_{\lambda}$ such that

$$
\left|\lambda-\frac{1}{m} \sum_{1 \leq j \leq m} \lambda_{j, h}\right| \leq c_{\lambda} \cdot h^{2} .
$$

The eigenvalues of $(\mathrm{E})$ that do not converge towards an eigenvalue of $(\mathrm{P})$ are zero and their number is $N_{s}$, the dimension of $W_{h}$.

Proof. We first prove that elements $E$ of $E_{\alpha}$ belong to $H^{2}$. Such an $E$ satisfies

$$
A\left((A-\alpha I)^{n_{\alpha}-1} E\right)=\alpha(A-\alpha I)^{n_{\alpha}-1} E,
$$

so $\left((A-\alpha I)^{n_{\alpha}-1} E\right)$ belongs to $H^{2}$, since $A$ goes from $L^{2}$ to $H^{2}$. We repeat the same process to conclude that $E \in H^{2}$ and $\operatorname{div} E \in L^{2}$. Then we have

$$
\begin{aligned}
\left\|A E-A_{h} E\right\|_{X}+\left\|B E-B_{h} E\right\|_{W} & \leq c \cdot h \cdot\left(\|A E\|_{2}+\|B E\|_{2}\right) \\
& \leq c \cdot h \cdot\left(\|E\|_{0}+\|\operatorname{div} E\|_{0}\right) .
\end{aligned}
$$


Since $\operatorname{dim} E_{\alpha}$ is finite, the $H$ (div) norm and $L^{2}$ norm are equivalent, so we have

$$
\left.\|\left(A-A_{h}\right)_{E_{\alpha}}\right)\left\|_{H X}+\right\|\left(B-B_{h}\right)_{E_{\alpha}} \|_{H W} \leq c_{\alpha} \cdot h .
$$

The same result holds for $A^{*}, B^{*}$.

The eigenvalues $\lambda$ of $(\mathrm{P})$ (resp. (E)) can be written as $(2-\alpha) / \alpha$, where $\alpha$ is an eigenvalue of $A$ (resp. $\left(A_{h}\right)$ ). Applying the theorem to (II)-(II.a), we translate the inequality to find the result for $(\mathrm{P})-(\mathrm{E})$. The gradients of $W_{h}$ 's functions are eigenvectors of the problem $(\mathrm{E})$ associated with the zero eigenvalue, but they have no meaning for the continuous problem, since they are not divergence-free.

Remark. We notice that these parasitic eigenvalues do not mix with our approximate ones; this is the advantage of the mixed method, which does not introduce any uncontrollable eigenvalue into the set. The existence of parasitic values is common when using conforming finite elements; such eigenvalues are often called phenomena of parasite modes.

\section{DISCONNECTED BOUNDARIES}

Now we will deal with the presence of kernels: when the boundary is not connected, there exist functions in $V$ that are curl-free. We want to study their approximate analogues more precisely.

We consider a polyhedral open set $\Omega$, bounded by

$$
\partial \Omega=\Gamma_{0}+\sum_{i=1, \ldots, p} \Gamma_{i} .
$$

Here, $\Gamma_{0}$ is the boundary of the unbounded component of $R^{3}-\Omega$. The $\Gamma_{i}, i=$ $1, \ldots, p$, are the other connected components. This is the case when a cavity has metallic kernels inside. The space of curl-free functions such that div $\varepsilon E=$ 0 , denoted by $H_{n}$, is no longer reduced to the element $E=0$. Specifically, we have the following

Lemma 4.1. The dimension of $H_{n}$ is exactly $p$.

Proof. In Bendali, Dominguez, and Gallic [2], we find the proof of this result and a construction of a basis in the case of the Laplacian (i.e., $\varepsilon=1$ ). We adapt easily their steps: to each kernel $i$, we associate $q_{i}$, the solution of the following problem:

$$
\left\{\begin{aligned}
\operatorname{div} \varepsilon \operatorname{grad} q_{i} & =0 & & \text { in } \Omega, \\
q_{i} & =0 & & \text { on } \Gamma_{0}, \\
q_{i} & =\delta_{i, j} & & \text { on } \Gamma_{j} .
\end{aligned}\right.
$$

Then $\operatorname{grad} q_{i}$ belongs to $H_{n}$, and $\left(\operatorname{grad} q_{i}, i=1, \ldots, p\right)$ forms a basis of this space.

We now introduce the new space

$$
W=\left\{q \in H^{1}(\Omega),\left.q\right|_{\Gamma_{0}}=0,\left.q\right|_{\Gamma_{i}} \equiv c_{i}\right\} .
$$

The space $X$ is the same as before, except for the definition of the boundary, which now includes the boundaries of the kernels. As $\Gamma_{i}$ is a level surface of $q, \operatorname{grad} q$ is orthogonal to $\Gamma_{i}$, and thus belongs to $X$. We consider the 
same bilinear forms $a, b, r$ as before for the saddle-point formulation of the problem: $a$ is elliptic on $V$; the Brezzi-Babuška condition is still satisfied, because the Poincaré inequality is still true for functions which are zero on part of the boundary. The property $(b(v, q)=0, \forall v \in X \Rightarrow q=0)$ also holds, since $\operatorname{grad} q$ belongs to $X$ and Poincaré's inequality implies $q=0$.

The saddle-point continuous formulation can be treated as in $\S 2$, and we have to add a zero eigenvalue for each metallic kernel in order to find the whole set of solutions for the problem $(\mathrm{P})$.

For the approximate formulation, we need to change a few details. We define $W_{h}$ as we did $W$, with $q$ constant on each $\Gamma_{i}$. And $X_{h}$ is also defined as in $\S 2$. This definition implies $\operatorname{grad} q$ belongs to $X_{h}$, for then $\operatorname{grad} q \times n=0$ on each $\Gamma_{i}$. Thus, we find the same properties satisfied by $a, b, r$ as for the continuous and approximate formulations in the case of a connected boundary: the nonzero eigenvalues converge with second-order precision, since we can apply the same theorems, with a small modification in the proof of Nedelec [9] owing to the boundary condition no longer being $\left.q\right|_{\Gamma_{i}} \equiv c_{i}$.

The gradients of $H_{0}^{1}$-functions of $W_{h}$ generate $N_{s}$ zero eigenvalues, and we still have to study the approximate analogues of the curl-free functions in $V$.

Theorem 4.2. Let $N_{c}$ be the number of metallic kernels inside the cavity. Then for $h$ small enough there are exactly $N_{s}+N_{c}$ zero eigenvalues for the approximate problem $(\mathrm{E})$. Since $\operatorname{grad}\left(W_{h}\right)$ is not included in $V, N_{s}$ eigenvectors are not in $V$. The $N_{s}$ associated eigenvalues are meaningless for the continuous problem $(\mathrm{P})$. The $N_{c}$ other zero eigenvalues are the numerical equivalents of the $N_{c}$ zero eigenvalues of the problem $(\mathrm{P})$, and there is no need to study their convergence rates.

Proof. Let $\prod q_{i}$ be the approximation of $q_{i}$ in $W_{h}$. Then $\prod q_{i}$ is curl-free (it is a gradient); furthermore, it is in $X_{h}$, and it does not belong to $\operatorname{grad} \prod\left(H_{0}^{1}\right)$. Indeed, suppose $\operatorname{grad} \Pi q_{i}=\operatorname{grad} p$ with $p$ in $H_{0}^{1} ;$ then $\operatorname{grad}\left(\Pi q_{i}-p\right)=0$. Let $M$ be a vertex on $\Gamma_{0}$ and $N$ a vertex on $\Gamma_{i}$; then

$$
\begin{aligned}
0 & =\int_{M}^{N} \operatorname{grad}\left(\prod q_{i}-p\right) \cdot \vec{d} s \\
& =\left(\prod q_{i}-p\right)(N)-\left(\prod q_{i}-p\right)(M)=\left(\prod q_{i}\right)(N)=1,
\end{aligned}
$$

which makes no sense. This implies $\operatorname{grad} \prod q_{i}$ has a projection on $V$ which is not null and is curl-free (the difference between two gradients). For each $\Gamma_{i}$, we have constructed an eigenvector for the approximate problem associated with the zero eigenvalue.

In short, we have

Theorem 4.3. Let $N_{c}$ be the number of metallic kernels inside the cavity, and let $N_{s}$ be the number of vertices of the triangulation inside. The eigenvalues of the approximate problem (E) can be divided into three sets

(1) $N_{s}$ zero eigenvalues not corresponding to the continuous problem $(\mathrm{P})$, since they are not divergence-free. (They are introduced by the finite element space. But we know their number, so we can easily eliminate them.)

(2) $N_{c}$ zero eigenvalues corresponding exactly to the $N_{c}$ zero eigenvalues of $(\mathrm{P})$. 
(3) $N$ nonzero eigenvalues ( $\left.N=\operatorname{dim}\left(X_{h}\right)-N_{s}-N_{c}\right)$ that converge with second-order rate toward the nonzero eigenvalues of $(\mathrm{P})$.

\section{NUMERICAL EXPERIMENTS}

To illustrate our theory, we consider a cubic cavity, $L=25$ centimeters long, filled with a homogeneous medium $\varepsilon=\mu=1$. In this case, the eigenvalues are easy to calculate. For each triplet $(m, n, p)$ in $N \times N \times N$, of which at least two are positive integers, we have the eigenvalue

$$
k^{2}=\left(\frac{\pi}{L}\right)^{2}\left(m^{2}+n^{2}+p^{2}\right) .
$$

If the three integers are positive, the multiplicity of this eigenvalue associated with the triplet is two. Otherwise, the multiplicity is one, but an eigenvalue can be generated by more than one triplet.

One problem caused by the existence of zero eigenvalues in the approximate problem is the calculation of the first eigenvalues. A power method cannot be used, since the kernel is not empty, and we have to calculate the whole set of eigenvalues, which takes time.

In order to point out the decrease of the eigenvalues, we use four different meshes: first, 3 vertices in each direction, then $4,5,6$. For each simulation, we erase $N_{s}(=1,4,27,125)$ zero eigenvalues in the approximate spectrum that come from the mixed elements. Then we trace the decrease of the first nineteen eigenvalues, $h=L / 2, \ldots, L / 5$. In order to facilitate the interpretation of these curves, we trace a reference curve $y=a \cdot x-2$, and we use logarithmic scales. We can see the global tendency of decreasing with second-order rate.

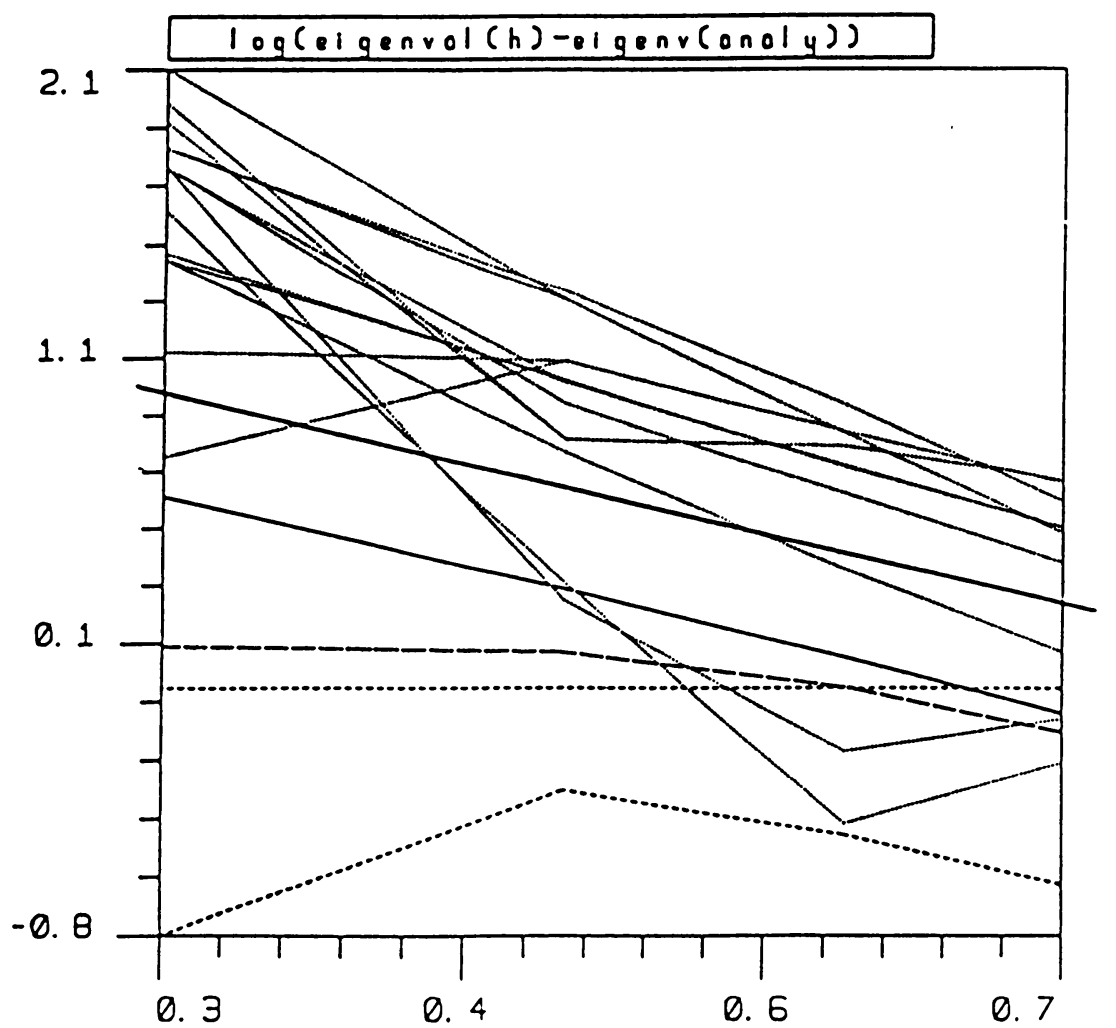

$\log (1 / h)$ 


\section{Conclusion}

We have selected polyhedral open sets to prevent the long calculation arising from the approximation of the set, and to apply easily the results of Nedelec [9]. But more recent work by Dubois [6] shows that the norms $\left\|A-A_{h}\right\|,\left\|B-B_{h}\right\|$ also decrease with first-order rate when the boundary is regular enough and when curved finite elements are used. Thanks to this result, our study still holds when the open set is not polyhedral.

The main advantage of using mixed finite elements is to avoid the mixing of eigenvalues, a disadvantage of conforming finite elements. We find the analogues of the zero eigenvalues of the continuous problem by a subtraction: the number of zero eigenvalues minus the number of vertices inside the approximate set.

Finally, the convergence occurs with a second-order rate for the nonzero eigenvalues, and the convergence is trivial for the zero eigenvalues, when the boundary is not connected.

\section{BIBLIOGRAPHY}

1. S. Agmon, A. Douglis, and L. Nirenberg, Estimates near the boundary for solutions of elliptic partial differential equations satisfying general boundary value conditions. I, Comm. Pure Appl. Math. 12 (1959), 623-727.

2. A. Bendali, J. M. Dominguez, and S. Gallic, $A$ variational approach for the vecor potential formulation of the Stokes and Navier-Stokes problems in three dimensional domains, J. Math. Anal. Appl. 107 (1985), 537-560.

3. A. Bossavit, Whitney forms: a class of finite elements for three-dimensional computations in electromagnetism, IEEE Proc.-A 135 (1988), 493-500.

4. F. Brezzi, On the existence, uniqueness and approximation of saddle-point problems arising from Lagrangian multipliers, RAIRO (1974), 129-151.

5. F. Brezzi and P. A. Raviart, Mixed finite element methods for 4 th order elliptic equations, Topics in Numerical Analysis III (J. J. H. Miller, ed.), Academic Press, New York, 1977, 33-56.

6. F. Dubois, Discrete vector potential of a representation of a divergence-free vector field in three-dimensional domains: numerical analysis of a model problem, SIAM J. Numer. Anal. 27 (1990), 1103-1141.

7. K. Ishihara, The buckling of plates by the mixed finite element method, Mem. Numer. Math. 5 (1978), 73-82.

8. B. Mercier, J. Osborn, J. Rappaz, and P. A. Raviart, Eigenvalue approximation by mixed and hybrid methods, Math. Comp. 36 (1981), 427-453.

9. J. C. Nedelec, Mixed finite elements in $R^{3}$, Numer. Math. 35 (1980), 315-341.

10. J. Osborn, Spectral approximation for compact operators, Math. Comp. 29 (1975), 712-725.

Centre de Mathematiques Appliquées, Unite de Recherche Associée au CNRS-756, Ecole Polytechnique, 91128 Palaiseau Cedex, France

E-mail address: levillai@cmapx.polytechnique.fr 